\title{
Novel epigenetic signature of DNA damage response genes for prognostication and immunophenotype of non-G-CIMP glioblastomas
}

Bowen Li

Air Force Medical University

Fangfang Liu

Air Force Medical University

Weihong Hu

Air Force Medical University

Rui Li

Air Force Medical University

Amandine Etcheverry

Rennes 1 University: Universite de Rennes 1

Marc Aubry

Rennes 1 University: Universite de Rennes 1

Jean Mosser

Rennes 1 University: Universite de Rennes 1

Xiang Zhang

Air Force Medical University Xijing Hospital: Xijing Hospital

Yuanming Wu

Air Force Medical University

Anan Yin ( $\nabla$ yinanan@aliyun.com )

Xijing Hospital https://orcid.org/0000-0002-1888-5551

Kun Chen

Air Force Medical University

Yalong He

Air Force Medical University Xijing Hospital: Xijing Hospital

\section{Research}

Keywords: Glioblastoma, glioma-CpGs island methylator phenotype, Immunophenotype, DNA damage response, DNA methylation

Posted Date: August 23rd, 2021 
DOI: https://doi.org/10.21203/rs.3.rs-823543/v1

License: (c) (1) This work is licensed under a Creative Commons Attribution 4.0 International License. Read Full License 


\section{Abstract \\ Background}

Dysregulation and dysfunction of DNA damage response (DDR) have prognostic and predictive implications for glioblastomas (GBMs) without glioma-CpGs island methylator phenotype (G-CIMP); mathematical modeling based on DNA methylation abnormality in DDR genes may serve as clinically useful biomarkers.

\section{Methods}

Independent cohorts of non-G-CIMP GBMs and IDH wild type (wt) lower-grade gliomas (LGGs) from local and public databases were included for discovery and validation of a multimarker signature, combined using a RISK score model. Different bioinformatic and functional experiments were performed for biological validation.

\section{Results}

By analyzing DNA methylation microarray data of DDR genes, we totally identified five $\mathrm{CpGs}$, each of which was significantly correlated with overall survival (OS) of non-G-CIMP GBMs, independent of age, treatments and the 0-6-methylguanine-DNA methyltransferase (MGMT) methylation status. A RISK score signature of the $5 \mathrm{CpGs}$ was constructed and validated to powerfully and independently prognosticate prognosis in non-G-CIMP GBMs. It also showed good discriminating value in stratified cohorts by patient age and MGMT methylation status. Bioinformatic analysis revealed a close correlation of the DDR epigenetic signature to distinct immunophenotypes of non-G-CIMP GBMs. Functional studies showed that NSUN5, epigenetically regulated by one identified CpGs, exhibited tumor-suppressor characteristics but may have immunosuppressive implications and confer TMZ resistance to GBM cells.

\section{Conclusions}

The epigenetic signature of DDR genes might be of promising value for refining current prognostic classification of non-G-CIMP GBMs, and its potential links to distinct immunophenotypes make it a promising biomarker candidate in the coming era of cancer immunotherapy.

\section{Introduction}

Glioblastomas (GBMs) are the most frequent and devastating glioma subtype, and are refractory to the genotoxic therapies of radiotherapy (RT) and temozolomide (TMZ)[1]. Exposure to these genotoxic insults can generate DNA damage and pose threats to genome integrity and survival of GBM cells[2-4]. The incurability of GBMs is likely attributable to its profound DNA repair machinery to counteract those 
threats, but is hardly predictable due to the genomic and cellular heterogeneity[5]. Comprehensive characterization of molecular components in DNA damage response (DDR) may provide useful information on glioma progression.

By employing genome-scale CRISPR-based genetic screens, recent work has presented a global view of DDR genes, whose loss caused sensitization or resistance to DNA-damaging agents in a model of human cells[4]. Dysregulation and dysfunction in such DDR genes have implications for tumor progression, therapeutic resistance and anti-tumor immunity[2-4]. Mathematical modeling on molecular data of the DDR genes may serve as promising biomarkers with high clinically informative value.

Among all molecular machinery, DNA methylation represents one critical layer of control of gene activity and chromatin architecture[6]. Thanks to its distinctive physical-chemical and biological characters, DNA methylation has long been the leading candidate for cancer biomarkers discovery[6], such as methylation status of 0-6-methylguanine-DNA methyltransferase (MGMT) for prediction of TMZ benefit[7], and hypermethylation of glutathione S-transferase (GSTP1) for detection of prostate cancer[8]. Evaluation of DNA methylation abnormality in DDR genes can be informative but their biological and clinical implications have not been extensively studied in cancers including GBMs.

In this study, by analyzing genome-wide DNA methylation microarray data from independent cohorts with the major subgroup of GBMs that do not have the glioma-CpGs island methylator phenotype (G-CIMP), we established a novel 5-CpG signature located within the transcriptional regions of 5 DDR genes respectively; the epigenetic signature provided prognostic information that was complementary to current risk classifiers; the signature-defined risk subgroups were closely associated with distinct immunophenotypes. In vitro experiments on the epigenetically regulated gene NSUN5 provided biological evidence for the immune-relevant epigenetic signature of DDR genes.

\section{Materials And Methods}

\section{Molecular datasets from Rennes and Angers University}

A total of 125 primary non-G-CIMP GBMs were collected between 2004 and 2013 from the Neurosurgery Departments of Rennes and Angers University Hospitals (RAUH), including a new cohort of 77 samples (RAUH-new cohort[6]) profiled by Infinium HumanMethylation450k BeadChip (Illumina Inc.) and Agilent Whole HumanGenome $8 \times 60 \mathrm{~K}$ Microarray Kit (Agilent Technologies); and a published cohort of 48 samples (RAUH-GSE22891[9]) profiled by Infinium HumanMethylation27k BeadChip (Illumina Inc.) and Agilent Whole HumanGenome $4 \times 44 \mathrm{~K}$ Microarray Kit (Agilent Technologies). All patients were treated with surgery followed by RT plus concurrent and adjuvant TMZ. Sample collection, histological review, clinical follow-up and microarray profiling were previously reported. All patients provided written informed consent, in accordance with the French regulations and the Helsinki Declaration. G-CIMP status was determined by k-means $(\mathrm{k}=3)$ clustering on the 1503 featured probes reported by Noushmehr et al[10]. MGMT methylation status was determined using a logistic regression model based oncg12434587 and 
cg12981137[11]. The gene expression subtypes were predicted by Binary tree classification prediction using the 840 classifiers reported by Verhaak et al[12].

\section{Molecular datasets from public databases}

Additional DNA methylation and gene expression microarray data were obtained from public databases, including the clinically annotated cohort from TCGA (TCGA-Brennan et al[13], RT/TMZ, n=219; RT alone, $\mathrm{n}=73$ ), and three cohorts from the Gene Expression Omnibus (GEO; https://www.ncbi.nlm.nih.gov/geo/): (1) GSE50923[14], RT/TMZ, n=49; (2) GSE60274[15], RT/TMZ, n=32; RT alone, n= 27; (3) GSE36278[16], unknown treatments, $n=52$. Moreover, DNA methylation microarray data of IDHwt LGGs were obtained from TCGA[17] (WHO grade II to III, various treatments, n=94), Chinese Glioma Genome Atlas (CGGA[18], WHO grade II to III, various treatments, n=30), GSE48462[19] (WHO III, RT/PCV, n=21; RT alone, $n=34$ ). For comparison, DNA methylation data of G-CIMP (or IDH mutant) tumors from TCGA[13] and CGGA[18], gene expression data of G-CIMP (or IDH mutant) tumors from TCGA[13], CGGA[20] and GSE16011[21], and molecular data of non-tumor brains (NTBs) from TCGA[13], CGGA[18, 20], GSE60274[15], GSE16011[21] and our RAUH datasets were all obtained. In addition, DNA methylation data of 26 NTBs from GSE63347[22] were also downloaded for comparison of TCGA samples. Patient information for each included database was summarized in Table $\mathbf{S 1 .}$

\section{Probe selection and RISK score construction}

Initial CpGs probe selection was performed by removal of probes not covered on both $27 \mathrm{k}$ and $450 \mathrm{k}$ platforms, those targeting $X$ and $Y$ chromosomes, and those relevant to single-nucleotide polymorphisms (SNPs). A compendium of 842 genes has been recently identified as DDR genes in a CPRISPR-based study by Olivieri $\mathrm{M}$ et al[4]. The 842 DDR genes were corresponded to the initially screened CpG probes based on gene symbols across PubMed gene database (https://www.ncbi.nlm.nih.gov/gene). A total of $1095 \mathrm{CpG}$ probes corresponding to 635 DDR genes were kept for further selection. To make DNA methylation microarray data comparable, batch effects between each platform and dataset were adjusted by M-value transformation and the empirical Bayes approach (ber R package)[23]. Missing $\beta$ values were imputed by impute R package. TCGA-Brennan et al and RAUH-new cohort were used for discovery phase. Selected $\mathrm{CpGs}$ with higher variability in methylation levels were used to correlate with OS by univariate Cox regression model and permutation test (Fig. 1). After the removal of inconsistent results from each discovery cohort, an overlap of $5 \mathrm{CpGs}$ were kept and subjected to multivariate Cox model within meta-discovery cohorts adjusted by age, MGMT methylation status and treatment. Finally, the 5-CpG panel was identified for constructing a RISK score model, which was the sum of $\beta$ values of each CpG weighted by their multivariate Cox coefficients, adjusted by age, MGMT methylation status, and treatment (Fig. 1). The cutoff for low-risk and high-risk tumors was predefined as median risk score from meta-discovery cohorts, or determined by maxstat $\mathrm{R}$ package[24].

\section{Gene set enrichment analysis}


Gene set enrichment analysis (GSEA) was run to evaluate functional profiles between grouped samples using the gene sets of the Gene Ontology Biological Processes and Kyoto Encyclopedia of Genes and Genomes (KEGG) from the Molecular Signatures Database (MSigDB)[25]. The proportion of 28 tumorinfiltrating immune cells (TIICs) in tumor bulks was estimated using single-sample GSEA (SSGSEA) algorithm and the 782-gene signature reported by Charoentong et al[26] (http://software.broadinstitute.org/gsea/msigdb/index.jsp). The abundance of the 28 TIICs in tumor samples was summarized as normalized enrichment scores (NES).

\section{Tumor mutation burden (TMB) calculation}

Mutation annotation format (MAF) flies for 212 non-G-CIMP GBMs were retrieved from the TCGA database (https://portal.gdc.cancer.gov/). The "maftools" R package was used to analyze the "Masked Somatic Mutation" type of somatic mutation data[27].

\section{Immunohistochemistry staining}

Formalin-fixed paraffin-embedded (FFPE) samples of 12 primary gliomas were collected from the Department of Neurosurgery, Xijing Hospital (NTBs, n=3; Grade II, n=3; Grade III, n=3; Grade IV, n=3) and were employed for immunohistochemistry (IHC) staining with anti-NSUN5 antibody (Abcam, ab121633). The percentages of positive cells were evaluated in at least five separate fields at $\times 400$ magnification. Immunoreactivity was scored as follows: 0 , no staining; 1 , weak staining in $<50 \%$ cells; 2 , weak staining in $\geq 50 \%$ cells; 3 , strong staining in $<50 \%$, cells; and 4 , strong staining in $\geq 50 \%$ cells[28].

\section{Cell culture and drugs}

Human glioma cell lines U251 and A172, obtained from American Type Culture Collection (ATCC) were cultured in DMEM (Sigma) containing $10 \%$ fetal bovine serum (FBS) respectively at $37^{\circ} \mathrm{C}$ in $5 \% \mathrm{CO}_{2}$. Temozolomide (MedChemExpress, \#HY-17364) was dissolved in dimethyl sulfoxide (DMSO, SigmaAldrich) at a concentration of $100 \mathrm{mM}$ and stored at $-80^{\circ} \mathrm{C}$.

\section{Pyrosequencing}

Total DNA was extracted in using EZ DNA Methylation-Gold ${ }^{\mathrm{TM}}$ Kit (Beijing Tianmo). Pyrosequencing was performed by Pyromark Q96 ID platform and analyzed by PyroMark CpG software (Qiagen). The following primers were used: cg01251255-F: 5'-GTTTTGTAAGGYGTGTGTGTGAT-3'; cg01251255-R: biotin-5'- TCAAATTAACAAAAACTTAAAAACC-3'; and cg01251255-seq: 5'-TATTTGTAGGGGTAGGGAGT-3'.

\section{Western blot}

Cell lysates were performed in RIPA buffer contained protease inhibitor and phosphatase inhibitor (Roche). The primary antibodies against IRF3 ${ }^{S 396}$ (CST, \#4947, 1:1000), IRF3 (CST, \#4302S, 1:1000), P65 ${ }^{\text {S536 }}$ (CST, \#3033S, 1:1000), P65 (CST, \#4764S, 1:1000), STING ${ }^{S 366}$ (CST, \#50907, 1:1000), STING (Abcam, ab171850, 1:1000), TBK1 ${ }^{\text {S172 }}$ (CST, \#5483, 1:1000), cGAS (Sigma Aldrich, \#HPA031700, 1:1000), 
GAPDH (Proteintech, \#60004-1-Ig, 1:20000), NSUN5 (Abcam, \#a121633, 1:1000) were used according to the manufacturers' recommendations. Each immunoblot was done at least thrice and the signals were quantified using ImageJ software (Bethesda).

\section{RNAi transfection}

For NSUN5 knockdown in vitro, negative control (siCtrl), NSUN5 small interfering RNA (si-NSUN5\#1 and \#2) were obtained from Ribobio ${ }^{\mathrm{Tm}}$. siRNAs were transfected in GBM cells at the concentration of $1 \mu \mathrm{g} / \mathrm{ml}$. X-tremeGENE siRNA Transfection Reagent (Roche) was employed for establishing cells transfecting process according to the manufacturers' recommendations. The sequences of siRNA were as follows: siCtrl (negative control); si-NSUN5\#1: GACCTGCTCCGATGATGTA; si-NSUN5\#2: GCTACCATGAGGTCCACTA.

\section{Cell viability and TMZ cytotoxicity assay}

After transfection, U251 cells were seeded on 96 well plates (5000 cells per well). CCK-8 kit was used for cell viability and TMZ cytotoxicity detection. U251 cells were treated TMZ at the concentration of $7.5,15,30,60,120,240,480 \mu \mathrm{M}$ for $48 \mathrm{~h}$. CCK-8 reagent was added to wells ( $10 \mu \mathrm{l} /$ well) and incubated at $37^{\circ} \mathrm{C}$ for $1 \mathrm{~h}$. The absorbance at $450 \mathrm{~nm}$ was measured for calculating the IC50 of TMZ, and for evaluating cell viability at indicated time points.

\section{Cell cycle and apoptosis assay}

U251 cells were harvested and fixed in $75 \%$ ethanol at $4^{\circ} \mathrm{C}$ for $1 \mathrm{~h}$, to analyze intracellular DNA content. Fixed cell suspension $\left(1 \times 10^{6}\right.$ cells) was then washed twice and stained with Annexin V-fluorescein isothiocyanate (FITC)/ Propidium iodide (PI) apoptosis detection Kit (Becton, Dickinson and Company). The cells were subjected to flow cytometric analysis.

\section{Cell migration and invasion assay}

U251 cells were cultured in the upside of the transwell chamber (Corning), $700 \mu$ media with $10 \%$ FBS was added in the bottom of the wells. The chamber was lightly wiped with a cotton swab after cultivating for $24 \mathrm{~h}$. The migrated cells were fixed with $4 \%$ paraformaldehyde and then stained with crystal violet solution and counted under a microscope in three fields $(\times 200)$. The process for cell invasion assay was similar with migration assay but the transwell membranes were coated with Matrigel (Corning).

\section{RNA sequencing}

Total RNA was isolated from cells using Axypre ${ }^{\text {TM }}$ Multisource Total RNA Miniprep Kit (Axygen, \#365) following the manufacturer's procedure. The purity and quantity of RNA were measured by the Bioanalyzer 2100 and RNA 6000 Nano Lab Chip Kit (Agilent Technologies) with RIN number >7.0. cDNA libraries were constructed in using NEB Next Ultra Directional RNA Library Prep Kit (NEB), then used the Illumina sequencing technology on an Illumina novaseq 6000 platform. Differential expression analysis 
was performed with p-value $\leq 0.05$ and absolute value of log 2 fold change $\geq 1$ for significance. Volcano plots were prepared to depict fold-change differences in gene expression.

\section{Statistical analysis}

Differences in clinical and molecular features within each subgroup were tested by unpaired t test, MannWhitney test, Fisher's exact or Chi-square test. Overall survival (OS) was the time interval from the date of diagnosis or treatment to the date of death or last follow-up. Progression-free survival (PFS) was the time interval from the date of diagnosis or treatment to the date of progression defined by the Macdonald criteria or Response Assessment in Neuro-Oncology (RANO) criteria or the date of death or last followup $[29,30]$. Survival data were estimated by the Kaplan-Meier Method, and compared by log-rank test. Univariate and multivariate Cox regression analysis was used to evaluate the prognostic correlation and independence of each variable. Meta-analysis was performed by the inverse-variance method where application of either fixed- or random effect models was based on statistical heterogeneity, with p-value for chi-square test $\leq 0.05$ for significance. The prognostic performance was evaluated by time-dependent receiver operating characteristic (ROC) curve (survcomp R package)[31]. All the calculations were done with SPSS statistics (SPSS software Inc.) and R software, with two-side $p$ value $\leq 0.05$ for significance.

\section{Results}

\section{Identification of a 5-CpG signature corresponding to DDR genes for prognostication of non-G-CIMP GBMs}

According to the cross-match and multi-step selection, we identified a total of $5 \mathrm{CpGs}$ corresponding to 5 DDR genes from meta-discovery cohorts; each $\mathrm{CpG}$ significantly prognosticates OS of non-G-CIMP GBMs, independent of age, treatment and MGMT methylation status (Fig. 1). Among the panel, three CpGs were located within promoter-associated $\mathrm{CpG}$ island (CGI) regions and two $\mathrm{CpGs}$ were within CGI-relevant shore regions (Table 1). Those CpGs were not among the reported G-CIMP classifiers but seemed to be among the genomic $\mathrm{CpGs}$ affected by G-CIMP as four out of the five $\mathrm{CpG}$ s showed significant alterations in methylation levels between tumors of each G-CIMP status and NTBs (Fig. S1). 
Table 1

Characteristics of the five prognostic CpGs corresponding to DNA damage response genes

\begin{tabular}{|c|c|c|c|c|c|}
\hline Probe ID & $\begin{array}{l}\text { Relevant gene } \\
\text { symbol }\end{array}$ & Chr. & $\begin{array}{l}\text { Relation to } \\
\text { gene region }\end{array}$ & $\begin{array}{l}\text { Relation to CpGs } \\
\text { island a }\end{array}$ & $\begin{array}{l}\text { Multivariate Cox } \\
\text { coefficients }^{\text {a }}\end{array}$ \\
\hline cg24035962 & NCOA4 & 10 & TSS200 & Island & -1.288 \\
\hline $\operatorname{cg} 21275690$ & THOC6 & 16 & TSS1500 & Shore ${ }^{b}$ & -1.955 \\
\hline $\operatorname{cg} 01251255$ & NSUN5 & 7 & TSS200 & Island & -0.948 \\
\hline $\operatorname{cg} 27508071$ & C7orf26 & 7 & TSS1500 & Island & 1.219 \\
\hline $\operatorname{cg} 24747122$ & GNA13 & 17 & TSS1500 & Shore ${ }^{b}$ & 1.274 \\
\hline \multicolumn{6}{|c|}{ Chr = chromosome; TSS = transcriptional start site } \\
\hline \multicolumn{6}{|c|}{$\begin{array}{l}\text { a Cox coefficients were calculated from multivariate analysis incorporating age, MGMT methylation } \\
\text { status and assigned treatments in meta-discovery cohorts of TCGA-Brennan et al and RAUH-new } \\
\text { cohort. }\end{array}$} \\
\hline
\end{tabular}

The CpGs were combined by a RISK-score model, which was constructed as follows: RISK score $=(-1.288$ $\times \beta$ value of $c g 24035962)+(-1.955 \times \beta$ value of $c g 21275690)+(-0.948 \times \beta$ value of $c g 01251255)+$ $(1.219 \times \beta$ value of $c g 27508071)+(1.274 \times \beta$ value of $c g 24747122)$.

Using the median RISK score of meta-discovery cohorts as cutoff $(0.2664)$, patients were divided into lowrisk and high-risk groups, where low-risk patients were significantly associated with longer OS than highrisk ones (Fig. 2A). Prognostic performances in each discovery cohort were also reported in Fig. 2A. Similar results were yielded using the optimal cutoff (0.1664) calculated by maxstat (Fig. S2).

\section{Validation of the 5-CpG RISK score signature in independent cohorts}

By applying the $5-\mathrm{CpG}$ signature to the validation phase, we reported that low-risk patients appeared to have longer OS than high-risk ones whereas statistically significant results were not always yielded in each validation set (Fig. 2B). Pooled analysis using patient-level data and meta-analysis using cohortlevel data both revealed the statistically significant survival difference between the risk subgroups (Fig. 2B). The 5-CpG signature was also extended in cohorts of IDHwt LGGs (Fig. 2C). Using the median risk score (0.1617) as cutoff, patients from meta-cohorts (CGGA, TCGA and GSE48462 collectively) were divided into risk subgroups, where low-risk patients tended to have longer OS than high-risk ones (Fig. 2C). Pooled analysis yielded significant OS difference between the risk subgroups (Fig. 2C). Metaanalysis confirmed the marginally significant favorable OS in low-risk IDHwt LGGs (Fig. 2C). Similar findings were observed with the cutoff calculated by maxstat (Fig. S2). The 5-CpG signature was also validated in terms of PFS outcome in meta-cohorts of TCGA-Brennan et al, RAUH-new cohort and GSE36278 (Fig. S3). 


\section{Risk classification of the 5-CpG signature in clinically or molecularly stratified cohorts}

To further evaluate the prognostic performance, we applied the 5-CpG signature in stratified cohorts by MGMT status (methylated vs. unmethylated) and patient age ( $<$ vs. $\geq 65$ years old). In meta-discovery cohorts, the 5-CpG signature showed good discriminating value for prognosis among patients with each MGMT status and age subclass (Fig. 3). The discriminating ability was validated in meta-validation cohorts and in all available cohorts (Fig. 3). Similar results were also yielded using the optimal cutoff calculated by maxstat ( Fig. S4).

\section{The 5-CpG signature was an independent prognosticator for non-G-CIMP GBMs}

Within meta-discovery cohorts, univariate Cox regression analysis showed that patient age, MGMT methylation status, treatment and the 5-CpG signature were significantly correlated with OS (Table 2). Multivariate analysis incorporating all significant variables from univariate model demonstrated the 5$\mathrm{CpG}$ signature as an independent prognostic factor. Similar findings were observed within metavalidation cohorts (Table 2). The time-dependent ROC analysis revealed that the combination of the 5$\mathrm{CpG}$ signature with the two conventional risk factors could provide refined prognostic classification in non-G-CIMP GBMs (Fig. S5). Moreover, for those who underwent RT/TMZ, the 5-CpG signature was found to be superior to MGMT methylation status in predicting OS among older population (Fig. S5). 
Table 2

Univariate and multivariate Cox regression analyses in non-G-CIMP GBMs

Variables
Univariate Cox model

HR

$95 \% \mathrm{Cl}$
Multivariate Cox model

HR

value
$95 \% \mathrm{Cl}$ value

Meta-discovery cohorts $(n=369)$

\begin{tabular}{|c|c|c|c|c|c|c|}
\hline Patient age (increasing years) & 1.036 & $\begin{array}{l}1.023- \\
1.048\end{array}$ & $\dot{0} 001$ & 1.026 & $\begin{array}{l}1.013- \\
1.038\end{array}$ & $\dot{0} 001$ \\
\hline $\operatorname{KPS}(<70 \text { vs. } \geq 70 \text { yrs })^{a}$ & 1.861 & $\begin{array}{l}1.059- \\
3.271\end{array}$ & 0.031 & & & \\
\hline $\begin{array}{l}\text { The 5-CpG signature (median risk } \\
\text { score for low vs. high) }\end{array}$ & 0.633 & $\begin{array}{l}0.494- \\
0.811\end{array}$ & $<.001$ & 0.595 & $\begin{array}{l}0.462- \\
0.766\end{array}$ & $\dot{\delta} 001$ \\
\hline $\begin{array}{l}\text { MGMT methylation status } \\
\text { (unmethylated vs. methylated) }\end{array}$ & 1.828 & $\begin{array}{l}1.403- \\
2.381\end{array}$ & $\hat{0.001}$ & 1.765 & $\begin{array}{l}1.349- \\
2.309\end{array}$ & $\hat{0.001}$ \\
\hline $\begin{array}{l}\text { Gene expression subtype (non- } \\
\text { proneural vs. pronerual) }\end{array}$ & 1.129 & $\begin{array}{l}0.837- \\
1.522\end{array}$ & 0.429 & & & \\
\hline Treatment (RT alone vs. RT/TMZ) & 3.368 & $\begin{array}{l}2.518- \\
4.505\end{array}$ & $<.001$ & 2.557 & $\begin{array}{l}1.862- \\
3.511\end{array}$ & $\hat{0} .001$ \\
\hline $\begin{array}{l}\text { Bevacizumab at recurrence (no vs. } \\
\text { yes) }\end{array}$ & 1.501 & $\begin{array}{l}0.961- \\
2.344\end{array}$ & 0.074 & & & \\
\hline $\begin{array}{l}\text { Patient cohorts (RAUH-new cohort vs } \\
\text { TCGA-Brennan et al) }\end{array}$ & 0.578 & $\begin{array}{l}0.432- \\
0.773\end{array}$ & <. 001 & 0.654 & $\begin{array}{l}0.481- \\
0.889\end{array}$ & 0.007 \\
\hline \multicolumn{7}{|l|}{ Meta-validation cohorts $(n=209)$} \\
\hline Patient age (increasing years) & 1.018 & $\begin{array}{l}1.006- \\
1.031\end{array}$ & 0.004 & 1.020 & $\begin{array}{l}1.005- \\
1.035\end{array}$ & 0.009 \\
\hline $\begin{array}{l}\text { The 5-CpG signature (median risk } \\
\text { score for low vs. high) }\end{array}$ & 0.604 & $\begin{array}{l}0.440- \\
0.828\end{array}$ & 0.002 & 0.699 & $\begin{array}{l}0.492- \\
0.993\end{array}$ & 0.046 \\
\hline $\begin{array}{l}\text { MGMT methylation status } \\
\text { (unmethylated vs. methylated) }\end{array}$ & 2.190 & $\begin{array}{l}1.591- \\
3.015\end{array}$ & $\dot{0} 001$ & 2.340 & $\begin{array}{l}1.637- \\
3.344\end{array}$ & $\dot{0} 001$ \\
\hline $\begin{array}{l}\text { Gene expression subtype (non- } \\
\text { proneural vs. pronerual) }\end{array}$ & 1.436 & $\begin{array}{l}0.802- \\
2.569\end{array}$ & 0.223 & & & \\
\hline
\end{tabular}

RAUH = Rennes and Angers University Hospitals; TCGA = The Cancer Genome Atlas; G-CIMP = glioma$\mathrm{CpGs}$ island methylator phenotype; $\mathrm{GBM}=$ glioblastoma; $\mathrm{KPS}$ = Karnof sky performance score; $\mathrm{TMZ}$ = temozolomide; $\mathrm{RT}$ = radiotherapy;

${ }^{\text {a }}$ Data were not available for more than half of patients to be analyzed and were excluded for multivariate model whether it was statistically significant or not in univariate model 


\begin{tabular}{|c|c|c|c|c|c|c|}
\hline \multirow[t]{2}{*}{ Variables } & \multicolumn{3}{|c|}{ Univariate Cox model } & \multicolumn{3}{|c|}{ Multivariate Cox model } \\
\hline & $\mathrm{HR}$ & $95 \% \mathrm{Cl}$ & $\begin{array}{l}P \\
\text { value }\end{array}$ & HR & $95 \% \mathrm{Cl}$ & $\begin{array}{l}P \\
\text { value }\end{array}$ \\
\hline Treatment (RT alone vs. RT/TMZ) & 1.844 & $\begin{array}{l}1.190- \\
2.856\end{array}$ & 0.006 & 1.842 & $\begin{array}{l}1.176- \\
2.884\end{array}$ & 0.008 \\
\hline $\begin{array}{l}\text { Bevacizumab at recurrence (no vs. } \\
\text { yes) }\end{array}$ & 1.247 & $\begin{array}{l}0.669- \\
2.327\end{array}$ & 0.487 & & & \\
\hline $\begin{array}{l}\text { Patient cohorts (each validation } \\
\text { cohort) }\end{array}$ & - & - & 0.194 & & & \\
\hline \multicolumn{7}{|c|}{$\begin{array}{l}\text { RAUH = Rennes and Angers University Hospitals; TCGA = The Cancer Genome Atlas; G-CIMP = glioma- } \\
\text { CpGs island methylator phenotype; GBM = glioblastoma; KPS = Karnofsky performance score; TMZ = } \\
\text { temozolomide; RT = radiotherapy; }\end{array}$} \\
\hline \multicolumn{7}{|c|}{$\begin{array}{l}\text { a Data were not available for more than half of patients to be analyzed and were excluded for } \\
\text { multivariate model whether it was statistically significant or not in univariate model }\end{array}$} \\
\hline
\end{tabular}

\section{The DDR epigenetic signature was associated with distinct immunophenotypes of non-G-CIMP GBMs}

Within TCGA-Brennan et al, correlation with known clinical and molecular features showed that the risk subgroups were not apparently correlated with gender, age and treatment (Fig. 4A). However, the risk subgroups appeared to be correlated with 1) the gene expression subtypes with mesenchymal subtype being highly enriched in high-risk tumors; 2) MGMT methylation status with unmethylated tumors being slightly enriched in high-risk tumors; and 3) the five non-G-CIMP DNA methylation clusters reported by Brennan et al[13] with M4 cluster being enriched in low-risk tumors (Fig. 4A). The over-representation of mesenchymal subtype in high-risk tumors was also observed within non-TCGA samples (Fig. S6).

GSEA on TCGA transcriptome data revealed very distinct expression profiles between the risk subgroups. As expected, DNA damage-related gene sets were enriched in high-risk tumors including intrinsic apoptotic signaling pathway in response to DNA damage and regulation of DNA damage response signal transduction by p53 class mediator (Fig. 4B and Table S2). More interestingly, high-risk tumors were found to be highly enriched with gene sets relevant with multiple regulatory processes of immune response, and in particular negative regulatory ones (Fig. 4B and Table S2). The enrichment of immune gene sets was mostly observed in high-risk tumors from non-TCGA tumors (Table S2). To investigate whether the abundance of TIICs had impacts on the distinct immune-relevant transcriptional profiles, NESs of the 28 TIICs were compared between the risk subgroups. In TCGA-Brennan et al, the risk subgroups showed significant differences in TIICs infiltration patterns; high-risk patients were associated with significantly higher infiltration of most of the TIICs (21 out of 28) in tumor bulks (Fig. 4C). Similar findings were observed in non-TCGA samples (Fig. S6). In addition to TIICs infiltration, tumor immunologic features also included the expression profiles of immune checkpoint molecules, which 
represent an important intrinsic immune escape mechanism[32], and tumor mutation landscapes, which partially reflect the extent of tumor immunogenicity [32]. We found that high-risk tumors appeared to express more immune checkpoint molecules in TCGA-Brennan et al and non-TCGA samples respectively whereas the TMB was not significantly differed between the TCGA risk subgroups (Fig. 4D-E and Fig. S67).

\section{NSUN5 impacted on immune-relevant expression profiles of GBM cells}

To gain biological insights into the 5-CpG signature, we selected one $\mathrm{CpG}$ component (cg01251255) for further analysis. The single $\mathrm{CpG}$ was located at promoter-associated CGI region of NSUN5. Janin et al[33] have previously demonstrated that transcriptional level of NSUN5 was epigenetically regulated by CGI methylation in glioma cells. In this study, we found that the CpG was hypermethylated in grade II gliomas and its methylation was decreased with tumor grade (Fig. S8). Correspondingly NSUN5 was upregulated in grade IV gliomas (Fig. S8). IHC staining on gliomas of different grades confirmed the increased NSUN5 protein levels in grade IV gliomas (Fig. S8). Further analysis revealed that apparent differences in CpG methylation and NSUN5 expression were mostly observed among gliomas with each IDH (or G-CIMP) status; NSUN5 was relatively hypomethylated and transcriptionally upregulated in IDHwt (or non-G-CIMP) gliomas across different grades (Fig. S8).

RNA sequencing on U251 cells, a common IDHwt glioma cell line with relatively hypomethylated $\mathrm{CpG}$ and higher NSUN5 expression (Fig. S8), reported that knockdown of NSUN5 resulted in a total of 219 differentially expressed genes (DEGs; 154 downregulated genes and 65 upregulated genes; Fig. 5A and Table S3). Bioinformatic analysis showed that the DEGs and in particular the downregulated genes were mostly implicated in immune-relevant biological processes and especially for innate immune response (Fig. 5 and Fig. S9). Cyclic GMP-AMP synthase (cGAS)/stimulator of interferon genes (STING) pathway has been highlighted as a link of DNA damage to the induction of innate immunity in tumor-immune cell interactions $[2,3]$. Western blot showed that depletion of NSUN5 decreased the protein level of cGAS, attenuated the phosphorylation of STING, TBK1 and P65, but enhanced the phosphorylation of IRF3 (Fig. 5D), suggesting its roles in differentially activating downstream signaling of cGAS/STING pathway.

\section{NSUN5 exhibited tumor-suppressor characteristics but conferred TMZ resistance to GBM cells}

To determine the effects of NSUN5 on malignant behaviors of U251 cells, a series of in vitro experimental assays were conducted. Knockdown of NSUN5 by small interfering RNA increased the proliferation rates of U251 cells (Fig. 6A). Cell cycle analysis revealed that NSUN5 knockdown apparently increased the proportion of G2/M phase and slightly decreased the proportions of G0/G1 and S phases (Fig. 6B). Flow cytometric analysis confirmed that depletion of NSUN5 inhibited apoptosis in U251 cells (Fig. 6H). Moreover, NSUN5 knockdown enhanced the migration and invasion rates of U251 cells (Fig. 6C-D). Within datasets of non-G-CIMP GBMs and IDHwt gliomas of grade II to IV, lower NSUN5 expressions were found to be associated with favorable OS (Fig. 6E-F). These data indicated tumor-suppressor roles of NSUN5 in GBM cells. To investigate the effects of NSUN5 on TMZ sensitivity, the viabilities of U251 cells under the treatment of a series dose of TMZ were monitored. CCK-8 assays showed that NSUN5 knockdown 
significantly increased the TMZ concentration to reduce cell viability to $50 \%$ in U251 cells (Fig. 6G). Depletion of NSUN5 also decreased the apoptotic rates of U251 cells when exposed to TMZ (Fig. 6H). Western blot showed that NSUN5 knockdown was associated with decreased protein levels of $\mathrm{p} 53$ and p21 (Fig. 6I). These data suggested that NSUN5 may confer TMZ resistance to GBM cells through mechanisms that involved p53-p21 pathway.

\section{Discussion}

The combination of RT and TMZ has long been the standard of care for GBMs[34]. Both RT and TMZ act by damaging the DNA of tumor cells[5]. DNA damage has the potential to cause genome instability, disarrange chromosome architecture, hinder transcription and generate DNA replication barriers, which are partially lethal to tumor cells[3]. DDR counteracts these deleterious outcomes and acts to maintain genome integrity through activation of the sophisticated signaling networks that detect, signal, and repair DNA injuries[5]. The molecularly heterogeneous GBMs have variable responses to DNA injuries and acquire varying degrees of therapy resistance by differential activation of DDR, at the center of which is the multiple DNA repair pathways[35]. The ability to acquire resistance is the major cause for treatment failure[35]. However, dysregulation and dysfunction in one certain component or pathway is unlikely to compromise the deleterious ability due to the complementation and coordination of each DNA repair pathway[35]. Recent work has illustrated a global picture of genetic components in every step of the DDR[4]. Comprehensive evaluation of those DDR detectors, mediators and effectors may serve as potential biomarkers for precision medicine.

DNA methylation has long been the ideal biomarker candidate for cancers as it has many advantages over genetic- or expression-based information such as having reliable DNA samples, altered patterns that have stability, tolerance of non-tumor cell contamination, multi-level biological implications, and druginduced reversibility[36]. Cancers including GBMs are commonly accompanied with genome-wide DNA methylation alterations, characteristic of global hypomethylation at gene-poor DNA repeats and large hypomethylated blocks at gene-rich regions concurrent with mosaicked CGI hypermethylation[37, 38]. The epigenetic aberrations result in altered gene expression profiles, mostly with the silencing of tumor suppressors by CGI hypermethylation and the activation of oncogenes by non-CGI hypomethylation and gene-body hypermethylation[39]. Increasing studies have highlighted the transcriptional control of cancerspecific DNA methylation abnormality in some of the DDR genes, and biomarkers using such DNA methylation data have proven to be clinically informative[40]. In this study, we performed genome-wide DNA methylation analyses on by-far the most comprehensive architecture of DDR genes in non-G-CIMP GBMs. G-CIMP tumors were excluded from our analyses because those tumors, exclusively carrying IDH mutations, represented a small subtype $(\sim 10 \%)$ and showed a very distinct molecular background and clinical prognosis compared to non-G-CIMP ones $[10,16]$. Totally we identified 5 highly informative CpGs, each of which strongly prognosticates survival of non-G-CIMP GBMs, independent of age, MGMT methylation status and treatment. To coordinate the prognostic information of the $5 \mathrm{CpGs}$, a RISK score algorithm was employed to produce a multi-marker signature. Applying the signature to different cohorts showed that it was a general and independent prognostic biomarker for non-G-CIMP GBMs. Increasing 
evidence has highlighted the molecular and clinical similarity of IDHwt LGGs to non-G-CIMP GBMs, and supported the inclusion of IDHwt LGGs in the broad spectrum of GBM-related studies[17]. Therefore, the GBM-derived signature had been expanded to validation cohorts of IDHwt LGGs wherein it showed similar ability to classify patients with distinct prognosis. Together our DDR epigenetic signature may represent a promising prognosticator for diffuse gliomas that do not have G-CIMP or IDH mutations.

Treatment choice is highly complex for the subpopulations of GBMs with unmethylated MGMT tumors or older age[41]. The combinatory genotoxic therapies conferred very limited benefits for unmethylated tumors at the expense of high medical cost and drug toxicity[7]. The elderly and frail subpopulation is highly heterogeneous but commonly associated with overall poor physical status, presence of comorbidity, decreased tolerance of genotoxic therapies, and expectation shifts to better quality of life rather than long survival[41]. Therefore, to discovery biomarkers for refining the risk classification of such subpopulation may represent a straightforward approach for selecting appropriate patients who are likely to benefit from genotoxic therapies with minimal risk. In this study, our DDR epigenetic signature showed good performance in the subgroups with unmethylated tumors or with older age, wherein it was able to distinguish patients with distinct prognosis, and its combination with conventional risk classifiers could refine current prognostic classification. Therefore, we proposed that the incorporation of our DDR epigenetic signature to current risk classification scheme could be helpful for guiding clinical choice toward more specified patients.

To gain biological explanation on the prognostic nature of the DDR epigenetic signature, we evaluated the transcriptional profiles underlying the risk subgroups. In line with expectations, DDR-relevant gene sets were significantly elevated in high-risk tumors, indicating an enhanced resistance to therapy and partially contributing to the unfavorable outcomes. More intriguingly, bioinformatic analysis revealed apparent and consistent correlations of the DDR epigenetic signature to immune-relevant expression profiles; specifically, high-risk tumors showed elevated expressions of multiple regulatory processes of immune response and in particular those negative ones. The higher abundance of TIICs in high-risk tumors was also observed, which might be a major cause for the enhanced immune expression profiles in such subgroup. In this study, we also found that high-risk tumors expressed more immune checkpoint molecules to facilitate escape from immune killing in different cohorts. Taken all, the data suggested that the DDR epigenetic signature might be associated with distinct immunophenotypes of non-G-CIMP GBMs with high-risk tumors having an intense local immune response.

The central nervous system (CNS) is capable of orchestrating intense immune responses, rather than immunologically privileged[42]. Increasing studies have highlighted the determinant roles of immune phenotype in glioma prognosis and treatment response[6]. Immune-based therapy has been by far the most promising anti-tumor modality with unprecedented clinical benefits[43]. However, the response to immunotherapy has been highly variable due to the complexity of tumor-immune dynamics, and many patients failed to benefit[43]. Therefore there is an urgent need for discovery of clinically informative biomarkers that are able to reflect the immune landscapes of individual patients and to optimize the patient selection for better immunotherapy outcome[43]. In recent years, compelling evidences have been 
accumulated linking DDR aberrations to activation of anti-tumor immunity[2, 3, 43, 44]. The DDR dysfunction-induced genotoxic stress could generate clustered lesion and complex DNA damage that leads to accumulation of point mutations and indels[44]. Those genomic alterations are associated with increased tumor immunogenicity which would raise the chances for immune system to recognize those tumor cells with non-self antigens and eventually elicit an anti-tumor immune response[44]. Therefore, molecular characterization of DDR genes may serve as promising biomarkers for predicting tumor immune landscape and response to immunotherapy. It is also known that current immunotherapy will be effective for 'hot' tumors with a preexisting active immune response, whereas 'cold' tumors are insensitive to immunotherapy, which are lack of TIICs infiltration and immune response[45]. In our study, the epigenetic signature of DDR genes had been shown to consistently correlate with distinct immunophenotypes and in particularly high-risk tumors exhibited an enhanced immune expression profiles, indicating its potential value for predicting response to immunotherapy. Taken all, our study proposed the DDR epigenetic signature as one of such promising biomarkers, but prospective validation in randomized trials of immune-based therapy is needed for final conclusion.

The biological implications were further exemplified by NSUN5, one of the five DDR genes corresponding to our multi-marker signature. NSUN5 encodes a RNA methyltransferase for 5-methylcytosine and plays essential roles in brain development and function[33]. Preliminary evidence has been accumulated linking NSUN5 to tumor progression and treatment sensitivity[33, 46]. Janin et al[33] has reported that NSUN5 exhibited tumor-suppressor characteristics in both in vivo and in vitro glioma models but its inactivation was associated with improved clinical outcome. Further assays identified that CGI hypermethylationinduced NSUN5 silencing inhibited global protein synthesis by producing a hypomethylation event at the C3782 position of the 28S rRNA and drove an adaptive translational program to stress. The authors thus proposed that NSUN5 epigenetic silencing was a likely pathway used by glioma cells to survival themselves from hostile stress by shutting down unnecessary energy-consuming processes, preventing accumulation of misfolded or damaged proteins and initiating transcription of selected mRNAs that are essential for adaptive cell response[33]. However, the aforementioned hypothesis appears not enough to fully elucidate the apparent paradox of inactivation of a tumor suppressor being an indicator of favorable prognosis. Olivieri et al [4] has recently identified NSUN5 as a candidate DDR gene, whose loss caused sensitization to $\mathrm{KBrO3}$, an oxidizing and DNA-damaging agent. In our study, we expanded the tumorsuppressor characteristics of NSUN5 from inhibiting cell proliferation to inducing cell apoptosis, inhibiting cell cycle progression and attenuating the ability to migration and invasion. In addition, NSUN5 was found to confer enhanced TMZ resistance to GBM cells by pathways involving p53 and p21. More interestingly, RNA sequencing demonstrated the impacts of NSUN5 on the immune-relevant expression profiles and in particular the expressions of innate immune response components in GBM cells. The DNAsensing cGAS/STING signaling has been established to link DDR and tumor innate immunity[2, 3]. It has been proposed that CGAS/STING pathway has dichotomous roles in tumors, with downstream activation of NF-KB being mainly a driver of multiple oncogenic signals, and activation of IRF3 being mainly a mediator of anti-tumor immunity [47, 48]. In our study, NSUN5 knockdown was found to inhibit NF-kB activation through cGAS/STING pathway but to specifically enhance IRF3 activation, suggesting its role 
in shifting the CGAS/STING-mediated tumor-suppressive signals to the deleterious ones. It should be noted that all the established tumor-suppressor roles of NSUN5 were observed in glioma models that are free of immune response and genotoxic treatment[33]. Our data suggested that the enhanced chemoresistance and the immunosuppressive implications by NSUN5 may likely be responsible for its inactivation as an indicator of favorable prognosis in gliomas with competent immunity and genotoxic treatment, which still needs experimental validation.

Additional limitations existed in the present study. (a) The lllumina 450k array provided a dramatic increase in the genomic coverage of CpGs compared to the 27k array. However, only a limited number of samples were available with Illumina 450k data. To ensure that there were enough samples to employ a discovery-validation approach, we decided to limit CpGs to those that appeared on both the 27k and 450k arrays, at the expense of higher genomic coverage. (b) Our microarray-based signature was not available for clinical use due to the inconvenience and unavailability of the genome-wide detection technique in real clinical setting. (c) Only one CpG and the corresponding gene NSUN5 have been selected for preliminary biological validation. This strategy ignored the contributions of other epigenetic and genomic components to the multi-marker signature.

\section{Conclusion}

In conclusion, we proposed and validated an epigenetic signature of DDR genes as a promising biomarker for refining current prognostic classification of non-G-CIMP GBMs; the DDR epigenetic signature was closely associated with distinct immunophenotypes, highlighting its potential role in guiding precise immunotherapy; despite with tumor-suppressor characteristics, the immunosuppressive and chemo-resistant implications of NSUN5 provided biological evidence for the multi-marker signature.

\section{Abbreviations}

DDR: DNA damage response; GBMs: glioblastomas; G-CIMP: glioma-CpGs island methylator phenotype; OS: overall survival; MGMT: 0-6-methylguanine-DNA methyltransferase; IDHwt: IDH wild type LGGs: lower-grade gliomas RT: radiotherapy TMZ: temozolomide GSTP1: glutathione S-transferase; CGI: CpG island; DEGs: differentially expressed genes; cGAS: Cyclic GMP-AMP synthase; STING: stimulator of interferon genes; CNS: central nervous system; SNPs: single-nucleotide polymorphisms; GSEA: Gene set enrichment analysis; KEGG: Kyoto Encyclopedia of Genes and Genomes; TIICs: tumor-infiltrating immune cells NES: normalized enrichment scores; TMB: Tumor mutation burden; MAF: Mutation annotation format; IHC: immunohistochemistry; PFS: Progression-free survival; ROC: receiver operating characteristic

\section{Declarations}

\section{Ethics approval and consent to participate}


Informed consent was obtained for all participants from the Neurosurgery Departments of Rennes and Angers University Hospitals and from the Department of Neurosurgery, Xijing hospital.

\section{Consent for publication}

Not applicable.

\section{Availability of data and materials}

The datasets used and/or analysed during the current study are available

from the corresponding author on reasonable request or public databases; TCGA: https://tcgadata.nci.nih.gov; CGGA: http://www.cgga.org.cn/; GEO: https://www.ncbi.nlm.nih.gov/geo/.

\section{Conflicts of interest}

The authors declare that they have no competing interests.

\section{Funding}

This work was partially funded by grants from National Natural Science Foundation of China (No. 81402049, 81802486, 81671476, 31570906), China Postdoctoral Science Foundation (No.2019M653971), Shandong Province Natural Science Foundation (No.ZR2020QH0233), the key research and development plan in Shaanxi (No. 2019SF-059, 2020SF-204), the Key Innovative Project in Shaanxi (No. 2021ZDLSF02-02 ), the President Foundation of the 960th Hospital of PLA (No.2018QN04) and by Grants from the Brittany Region (France) et the FEDER (Europe).

\section{Authors' contributions}

Conception and design of the study : AAY, KC, and YLH. Provision of study material or patients : $A E, M A$, and JM. Acquisition and assembly of data : AAY, BWL, WHH and RL. Project administration, Software, methodology : YMW, AAY, KC, FFL and RL. Analysis and interpretation of results: AAY, BWL, and KC. Manuscript writing : All authors. Final approval of manuscript: All authors.

\section{Acknowledgements}

We gratefully acknowledge all the patients who agreed to participate in this study and to those who have provided their medical care. We gratefully acknowledge Dr. Menei, Dr. Le Reste, Dr. Vauleon, Dr. Quillien, and the Tumor Banks from Angers and Rennes for their constant support in the collection, processing and histological analysis of tumor samples. We also gratefully thank those who are willing to share valuable scientific data (Dr. Rose K Lai and Prof. Jill Barnholtz-Sloan). I (AAY) want to thank my newborn daughter (Jiayan Yin) and my wife (Dr. Yu Dong) for their great support. The results published here are in part based upon data generated by China Glioma Genome Atlas, The Cancer Genome Atlas, German Cancer Research Center, the Gene Expression Omnibus, and the research teams mentioned in this study. 


\section{References}

1. Tan AC, Ashley DM, Lopez GY, Malinzak M, Friedman HS, Khasraw M. Management of glioblastoma: State of the art and future directions. CA Cancer J Clin. 2020;70:299-312. 10.3322/caac.21613.

2. Pilger $D$, Seymour LW, Jackson SP. Interfaces between cellular responses to DNA damage and cancer immunotherapy. Genes Dev. 2021;35:602-18. 10.1101/gad.348314.121.

3. Reislander T, Groelly FJ, Tarsounas M. DNA Damage and Cancer Immunotherapy: A STING in the Tale. Mol Cell. 2020;80:21-8. 10.1016/j.molcel.2020.07.026.

4. Olivieri M, Cho T, Alvarez-Quilon A, Li K, Schellenberg MJ, Zimmermann M. A Genetic Map of the Response to DNA Damage in Human Cells. Cell. 2020;182:481-96. 10.1016/j.cell.2020.05.040.

5. Annovazzi L, Mellai M, Schiffer D, Chemotherapeutic Drugs. DNA Damage and Repair in Glioblastoma. Cancers (Basel). 2017; 9. 10.3390/cancers9060057.

6. Yin A, Shang Z, Etcheverry A, He Y, Aubry M, Lu N. Integrative analysis identifies an immune-relevant epigenetic signature for prognostication of non-G-CIMP glioblastomas. Oncoimmunology. 2021;10:1902071. 10.1080/2162402X.2021.1902071.

7. Hegi ME, Diserens AC, Gorlia T, Hamou MF, de Tribolet N, Weller M. MGMT gene silencing and benefit from temozolomide in glioblastoma. N Engl J Med. 2005;352:997-1003. 10.1056/NEJMoa043331.

8. Lee WH, Morton RA, Epstein JI, Brooks JD, Campbell PA, Bova GS. Cytidine methylation of regulatory sequences near the pi-class glutathione S-transferase gene accompanies human prostatic carcinogenesis. Proc Natl Acad Sci U S A. 1994;91:11733-37. 10.1073/pnas.91.24.11733.

9. Etcheverry A, Aubry M, de Tayrac M, Vauleon E, Boniface R, Guenot F. DNA methylation in glioblastoma: impact on gene expression and clinical outcome. BMC Genom. 2010;11:701. 10.1186/1471-2164-11-701.

10. Noushmehr H, Weisenberger DJ, Diefes K, Phillips HS, Pujara K, Berman BP. Identification of a CpG island methylator phenotype that defines a distinct subgroup of glioma. Cancer Cell. 2010;17:51022. 10.1016/j.ccr.2010.03.017.

11. Bady P, Sciuscio D, Diserens AC, Bloch J, van den Bent MJ, Marosi C. MGMT methylation analysis of glioblastoma on the Infinium methylation BeadChip identifies two distinct $\mathrm{CpG}$ regions associated with gene silencing and outcome, yielding a prediction model for comparisons across datasets, tumor grades, and CIMP-status. Acta Neuropathol. 2012;124:547-60. 10.1007/s00401-012-1016-2.

12. Verhaak RG, Hoadley KA, Purdom E, Wang V, Qi Y, Wilkerson MD. Integrated genomic analysis identifies clinically relevant subtypes of glioblastoma characterized by abnormalities in PDGFRA, IDH1, EGFR, and NF1. Cancer Cell. 2010;17:98-110. 10.1016/j.ccr.2009.12.020.

13. Brennan CW, Verhaak RG, McKenna A, Campos B, Noushmehr H, Salama SR. The somatic genomic landscape of glioblastoma. Cell. 2013;155:462-77. 10.1016/j.cell.2013.09.034.

14. Lai RK, Chen Y, Guan X, Nousome D, Sharma C, Canoll P. Genome-wide methylation analyses in glioblastoma multiforme. PLoS One. 2014;9:e89376. 10.1371/journal.pone.0089376. 
15. Kurscheid S, Bady P, Sciuscio D, Samarzija I, Shay T, Vassallo I. Chromosome 7 gain and DNA hypermethylation at the HOXA10 locus are associated with expression of a stem cell related HOXsignature in glioblastoma. Genome Biol. 2015;16:16. 10.1186/s13059-015-0583-7.

16. Sturm D, Witt H, Hovestadt V, Khuong-Quang DA, Jones DT, Konermann C. Hotspot mutations in H3F3A and IDH1 define distinct epigenetic and biological subgroups of glioblastoma. Cancer Cell. 2012;22:425-37. 10.1016/j.ccr.2012.08.024.

17. Ceccarelli M, Barthel FP, Malta TM, Sabedot TS, Salama SR, Murray BA. Molecular Profiling Reveals Biologically Discrete Subsets and Pathways of Progression in Diffuse Glioma. Cell. 2016;164:55063. 10.1016/j.cell.2015.12.028.

18. Zhang W, Yan W, You G, Bao Z, Wang Y, Liu Y. Genome-wide DNA methylation profiling identifies ALDH1A3 promoter methylation as a prognostic predictor in G-CIMP- primary glioblastoma. Cancer Lett. 2013;328:120-25. 10.1016/j.canlet.2012.08.033.

19. van den Bent MJ, Erdem-Eraslan L, Idbaih A, de Rooi J, Eilers PH, Spliet WG. MGMT-STP27 methylation status as predictive marker for response to PCV in anaplastic Oligodendrogliomas and Oligoastrocytomas. A report from EORTC study 26951. Clin Cancer Res. 2013; 19: 5513-22. 10.1158/1078 - 0432.CCR-13-1157.

20. Wang Y, Qian T, You G, Peng X, Chen C, You Y. Localizing seizure-susceptible brain regions associated with low-grade gliomas using voxel-based lesion-symptom mapping. Neuro Oncol. 2015;17:282-88. 10.1093/neuonc/nou130.

21. Gravendeel LA, Kouwenhoven MC, Gevaert O, de Rooi JJ, Stubbs AP, Duijm JE. Intrinsic gene expression profiles of gliomas are a better predictor of survival than histology. Cancer Res. 2009;69:9065-72. 10.1158/0008-5472.CAN-09-2307.

22. Horvath S, Garagnani P, Bacalini MG, Pirazzini C, Salvioli S, Gentilini D. Accelerated epigenetic aging in Down syndrome. Aging Cell. 2015;14:491-95. 10.1111/acel.12325.

23. Johnson WE, Li C, Rabinovic A. Adjusting batch effects in microarray expression data using empirical Bayes methods. Biostatistics. 2007;8:118-27. 10.1093/biostatistics/kxj037.

24. Hothorn T, Zeileis A. Generalized maximally selected statistics. Biometrics. 2008;64:1263-69. 10.1111/j.1541-0420.2008.00995.x.

25. Subramanian A, Tamayo P, Mootha VK, Mukherjee S, Ebert BL, Gillette MA. Gene set enrichment analysis: a knowledge-based approach for interpreting genome-wide expression profiles. Proc Natl Acad Sci U S A. 2005;102:15545-50. 10.1073/pnas.0506580102.

26. Charoentong P, Finotello F, Angelova M, Mayer $C$, Efremova M, Rieder D. Pan-cancer Immunogenomic Analyses Reveal Genotype-Immunophenotype Relationships and Predictors of Response to Checkpoint Blockade. Cell Rep. 2017;18:248-62. 10.1016/j.celrep.2016.12.019.

27. Mayakonda A, Lin DC, Assenov Y, Plass C, Koeffler HP. Maftools: efficient and comprehensive analysis of somatic variants in cancer. Genome Res. 2018;28:1747-56. 10.1101/gr.239244.118.

28. Yin AA, He YL, Etcheverry A, Liu YH, Aubry M, Barnholtz-Sloan J. Novel predictive epigenetic signature for temozolomide in non-G-CIMP glioblastomas. Clin Epigenetics. 2019;11:76. 10.1186/s13148-019- 
0670-9.

29. Macdonald DR, Cascino TL, Schold SJ, Cairncross JG. Response criteria for phase II studies of supratentorial malignant glioma. J Clin Oncol. 1990;8:1277-80. 10.1200/JC0.1990.8.7.1277.

30. Wen PY, Macdonald DR, Reardon DA, Cloughesy TF, Sorensen AG, Galanis E. Updated response assessment criteria for high-grade gliomas: response assessment in neuro-oncology working group. J Clin Oncol. 2010;28:1963-72. 10.1200/JC0.2009.26.3541.

31. Heagerty PJ, Lumley T, Pepe MS. Time-dependent ROC curves for censored survival data and a diagnostic marker. Biometrics. 2000;56:337-44.

32. Wang S, Zhang Q, Yu C, Cao Y, Zuo Y, Yang L. Immune cell infiltration-based signature for prognosis and immunogenomic analysis in breast cancer. Brief Bioinform. 2021;22:2020-31.

10.1093/bib/bbaa026.

33. Janin M, Ortiz-Barahona V, de Moura MC, Martinez-Cardus A, Llinas-Arias P, Soler M. Epigenetic loss of RNA-methyltransferase NSUN5 in glioma targets ribosomes to drive a stress adaptive translational program. Acta Neuropathol. 2019;138:1053-74. 10.1007/s00401-019-02062-4.

34. Yin AA, Cheng JX, Zhang X, Liu BL. The treatment of glioblastomas: a systematic update on clinical Phase III trials. Crit Rev Oncol Hematol. 2013;87:265-82. 10.1016/j.critrevonc.2013.01.007.

35. Ferri A, Stagni V, Barila D. Targeting the DNA Damage Response to Overcome Cancer Drug Resistance in Glioblastoma. Int J Mol Sci. 2020; 21. 10.3390/ijms21144910.

36. Yin AA, Lu N, Etcheverry A, Aubry M, Barnholtz-Sloan J, Zhang LH. A novel prognostic six-CpG signature in glioblastomas. CNS Neurosci Ther. 2018;24:167-77. 10.1111/cns.12786.

37. Esteller M. Epigenetics in cancer. N Engl J Med. 2008;358:1148-59. 10.1056/NEJMra072067.

38. Timp W, Bravo HC, McDonald OG, Goggins M, Umbricht C, Zeiger M. Large hypomethylated blocks as a universal defining epigenetic alteration in human solid tumors. Genome Med. 2014;6:61.

10.1186/s13073-014-0061-y.

39. Ehrlich M. DNA hypomethylation in cancer cells. Epigenomics. 2009;1:239-59. 10.2217/epi.09.33.

40. Uddin MS, Mamun AA, Alghamdi BS, Tewari D, Jeandet P, Sarwar MS. Epigenetics of glioblastoma multiforme: From molecular mechanisms to therapeutic approaches. Semin Cancer Biol. 2020. 10.1016/j.semcancer.2020.12.015.

41. Yin AA, Zhang LH, Cheng JX, Dong Y, Liu BL, Han N. Radiotherapy plus concurrent or sequential temozolomide for glioblastoma in the elderly: a meta-analysis. PLoS One. 2013;8:e74242. 10.1371/journal.pone.0074242.

42. Perng P, Lim M. Immunosuppressive Mechanisms of Malignant Gliomas: Parallels at Non-CNS Sites. Front Oncol. 2015;5:153. 10.3389/fonc.2015.00153.

43. Zhang J, Shih D, Lin SY. Role of DNA repair defects in predicting immunotherapy response. Biomark Res. 2020;8:23. 10.1186/s40364-020-00202-7.

44. Lamberti G, Andrini E, Sisi M, Federico AD, Ricciuti B. Targeting DNA damage response and repair genes to enhance anticancer immunotherapy: rationale and clinical implication. Future Oncol. 
2020;16:1751-66. 10.2217/fon-2020-0215.

45. Wu XN, Su D, Mei YD, Xu MQ, Zhang H, Wang ZY. Identified lung adenocarcinoma metabolic phenotypes and their association with tumor immune microenvironment. Cancer Immunol Immunother. 2021. 10.1007/s00262-021-02896-6.

46. Jiang Z, Li S, Han MJ, Hu GM, Cheng P. High expression of NSUN5 promotes cell proliferation via cell cycle regulation in colorectal cancer. Am J Transl Res. 2020;12:3858-70.

47. Ng KW, Marshall EA, Bell JC, Lam WL. cGAS-STING and Cancer: Dichotomous Roles in Tumor Immunity and Development. Trends Immunol. 2018;39:44-54. 10.1016/j.it.2017.07.013.

48. Kwon J, Bakhoum SF. The Cytosolic DNA-Sensing cGAS-STING Pathway in Cancer. Cancer Discov. 2020;10:26-39. 10.1158/2159-8290.CD-19-0761.

\section{Figures}




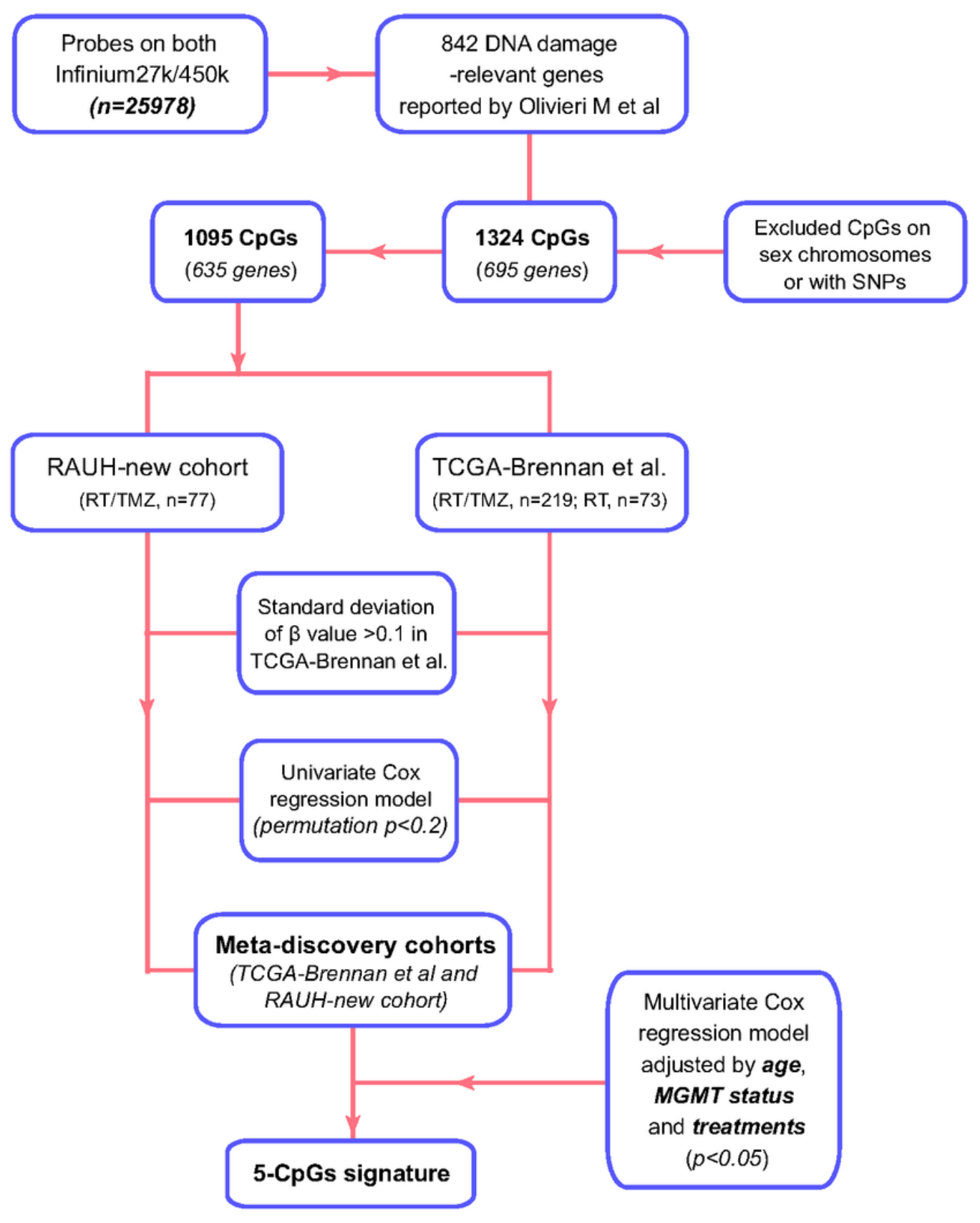

\section{Figure 1}

The study workflow regarding the selection of the prognostic CpGs corresponding to DDR genes in non-GCIMP GBMs G-CIMP=glioma $\mathrm{CpGs}$ island methylator phenotype; $\mathrm{GBM=glioblastoma;} \mathrm{DDR}=\mathrm{DNA}$ damage response 
A

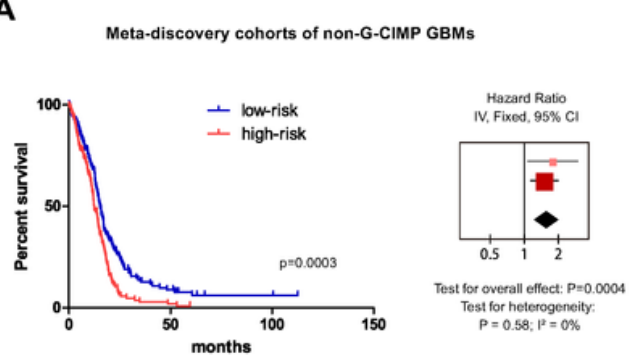

B

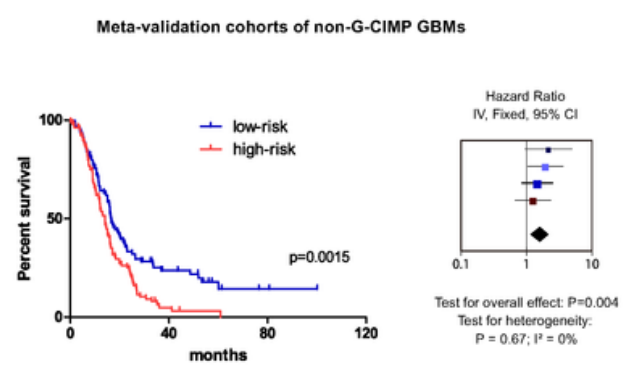

C
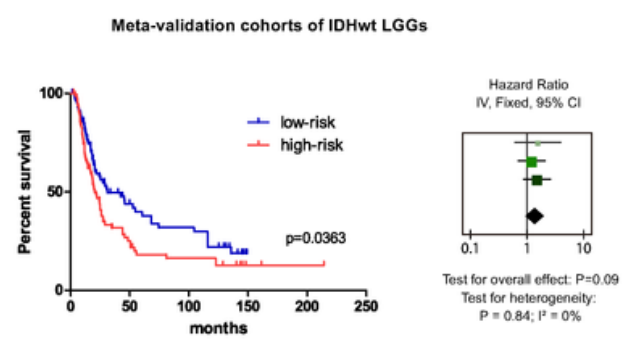

TCGA-Brennan et al

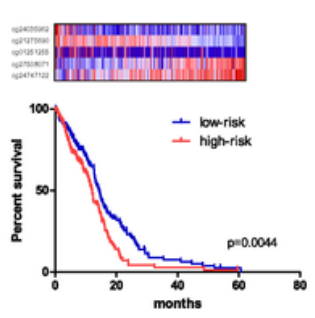

GSE50923
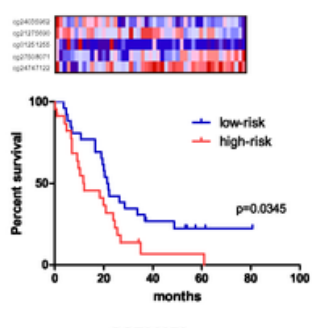

GSE36278

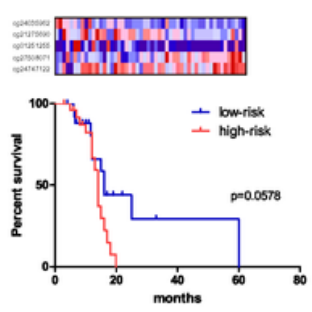

GSE48462

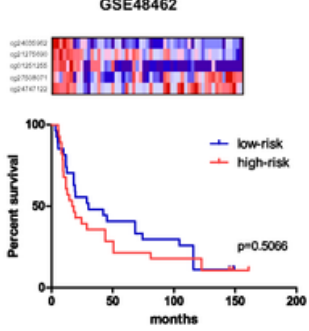

RAUH-new cohort

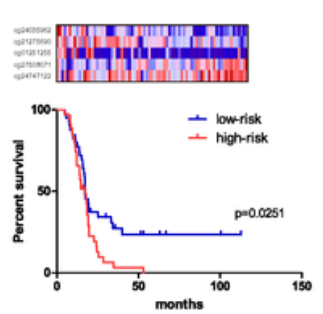

GSE60274
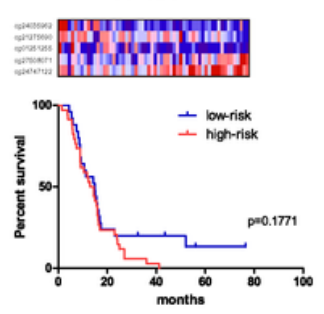

RAUH-GSE22891

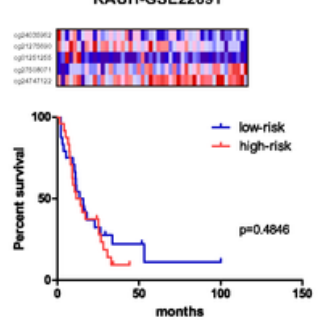

CGGA
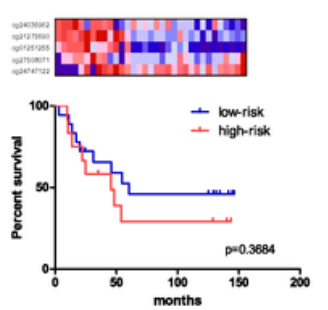

$\square$

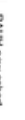



$\square$

量

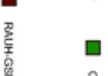

$\begin{array}{ll}\square & \square \\ 8 & \overrightarrow{0} \\ 0 & \end{array}$

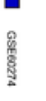

畹

\section{Figure 2}

The prognostic performance of the $5-\mathrm{CpG}$ signature in various cohorts The prognostic performance of the $5-\mathrm{CpG}$ signature in (A) discovery cohorts of non-G-CIMP GBMs, (B) validation cohorts of non-G-CIMP GBMs and (C) validation cohorts of IDHwt LGGs; survival curves of meta-cohorts (left) and each cohort (right), as well as forest plots of meta-analysis (middle) were presented; heatmaps of methylation levels of the five CpGs for each patient cohort were also shown; each row represented a CpGs; each column represented a sample which was ordered by the assigned risk scores; it was noted that the cutoff was predefined as the median risk score value from meta-discovery cohorts (TCGA-Brennan et al and RAUHnew cohort collectively); G-CIMP=glioma $\mathrm{CpGs}$ island methylator phenotype; $\mathrm{GBM=glioblastoma;}$ LGG=lower grade glioma 
A

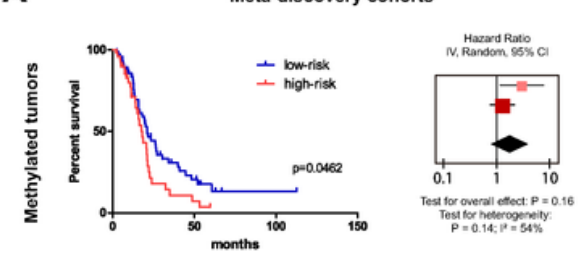

B

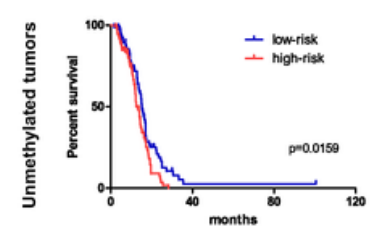

C
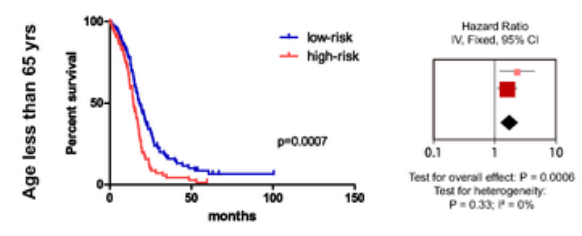

D

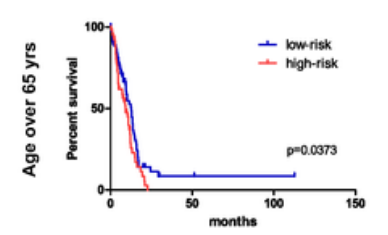

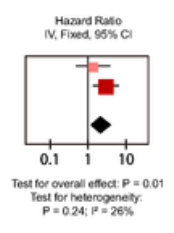
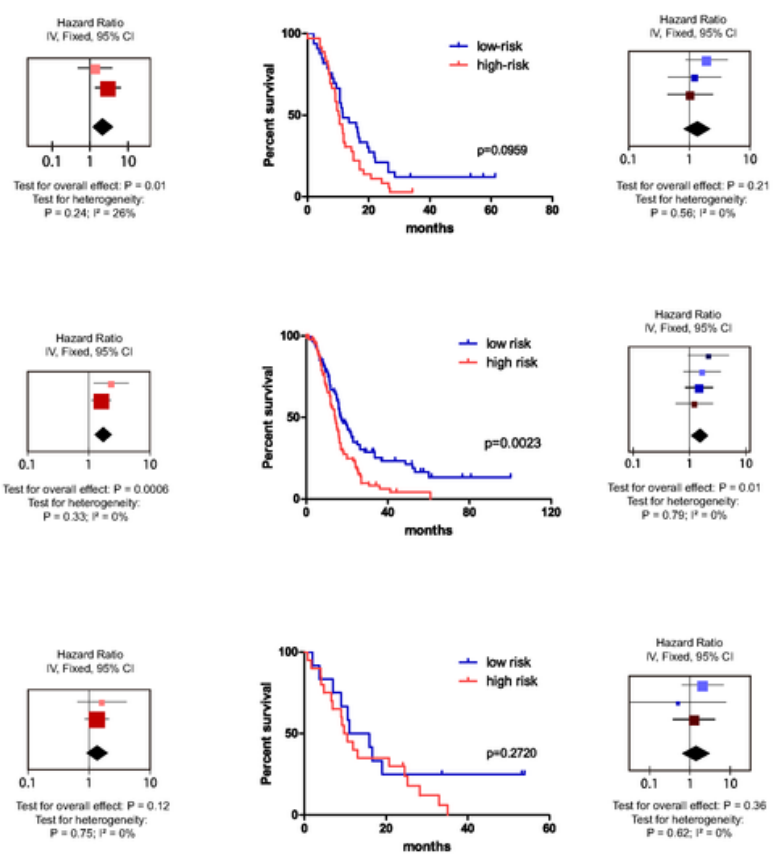

Meta-validation cohorts
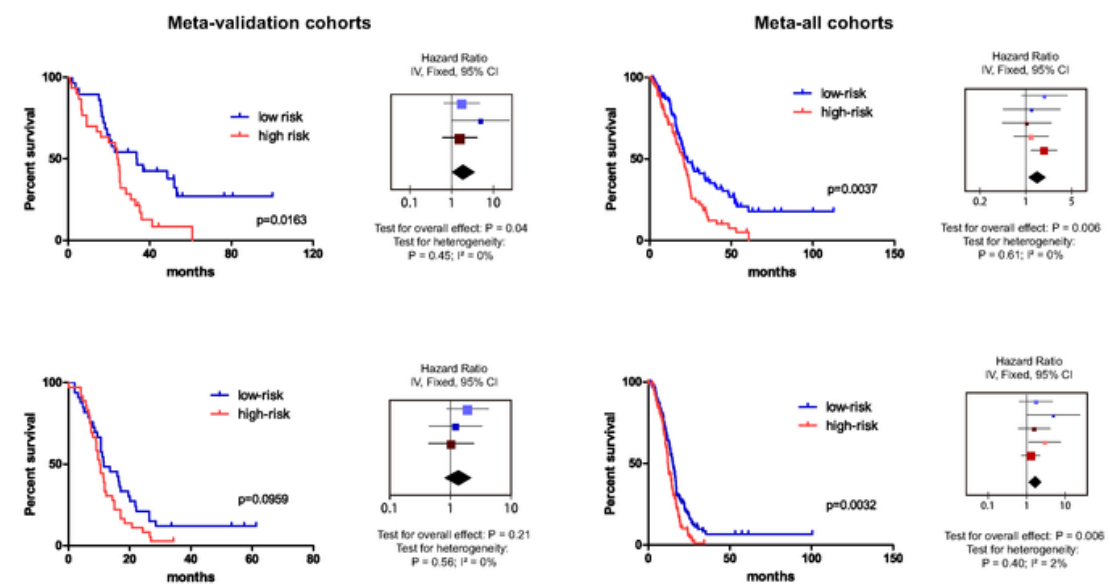

Maxard Rato
N. Fxed, 95\% CI
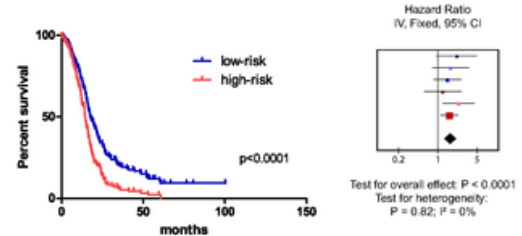

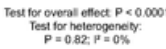

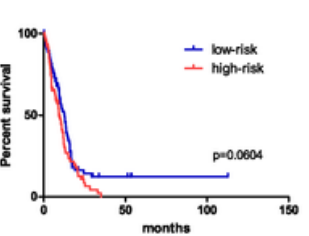

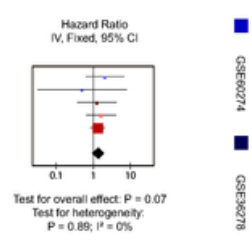

\section{Figure 3}

The prognostic performance of the 5-CpG signature in molecularly and clinically stratified cohorts Survival curves (left) and forest plots of meta-analysis (right) in each patient subgroup with (A) MGMT methylated tumors, (B) MGMT unmethylated tumors, (C) age < 65 years old and (D) age $\geq 65$ years old; it was noted that the cutoff was predefined as the median risk score value from meta-discovery cohorts (TCGA-Brennan et al and RAUH-new cohort collectively); MGMT=the 0-6-methylguanine-DNA methyltransferase 

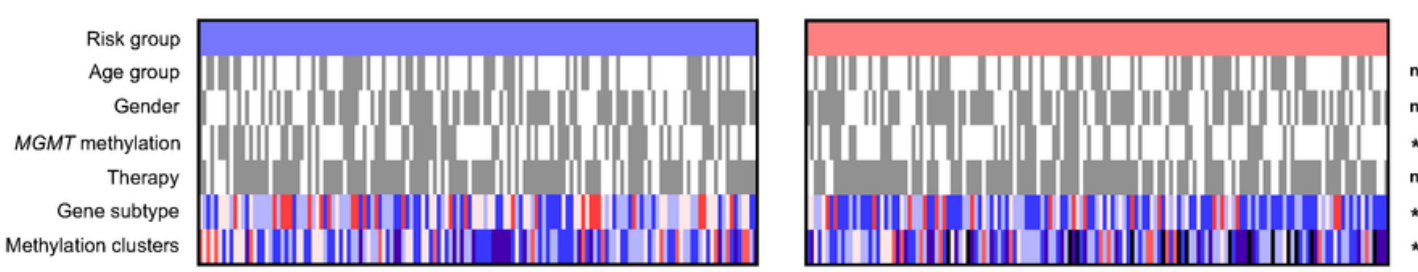

B


C
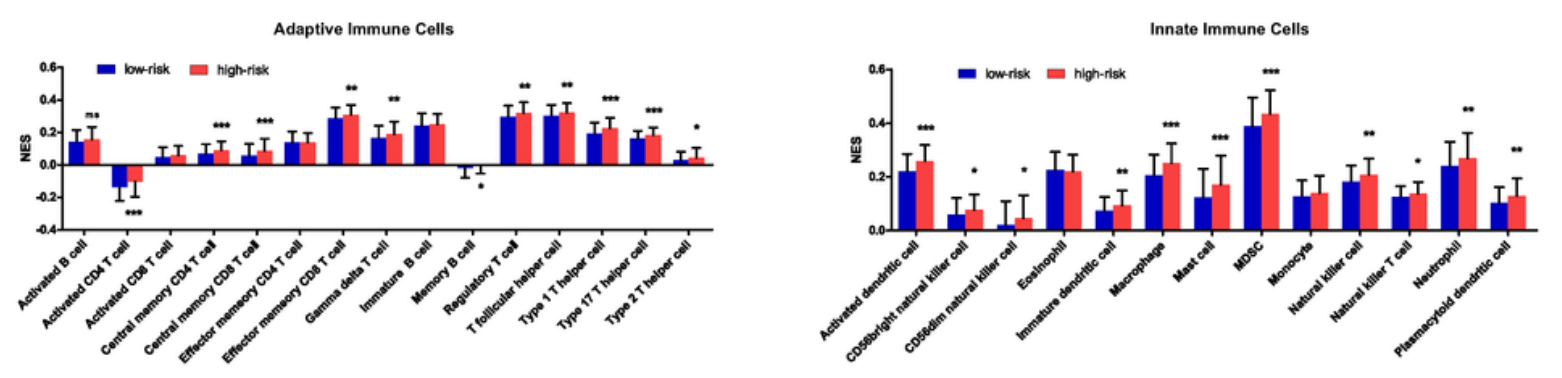

D


E

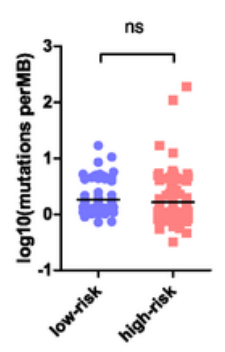

\section{Figure 4}

Molecular and clinical characterization of the $5-\mathrm{CpG}$ signature in TCGA samples (A) heatmaps of clinical and molecular features; each row represented a feature, and each column represented a sample, which was ordered by the assigned risk scores; (B) GSEA enrichment plots of representative gene sets for highrisk tumors; (C) The abundance of adaptive and innate immune infiltrating cells in low-risk vs. high-risk tumors; (D) Comparison of gene expression data (z-scores) of the immune checkpoint molecules between low-risk vs. high-risk tumors; (E) Comparison of TMB between low-risk vs. high-risk tumors from TCGA- 
Brennan et al; statistical significance at the level of $\mathrm{ns}>0.10, \mathrm{~ms}=0.05 \sim 0.10, *<0.05, * \star<0.01$ and $* \star \star$ $<0.001$; GSEA=Gene set enrichment analysis; TCGA=The Cancer Genome Atlas; TMB=tumor mutation burden

A



D

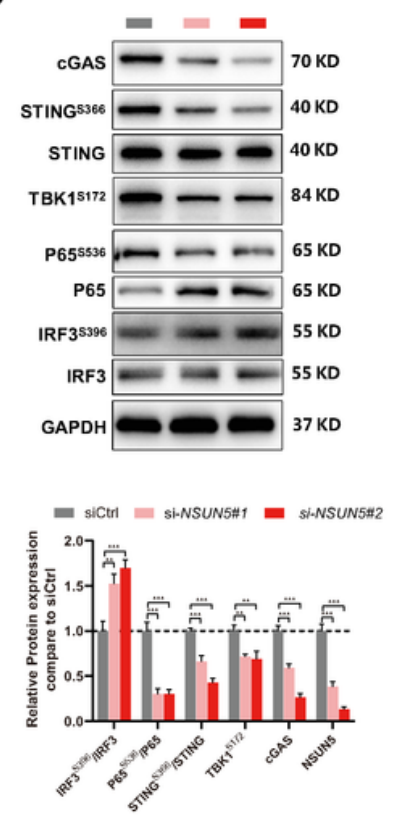

B

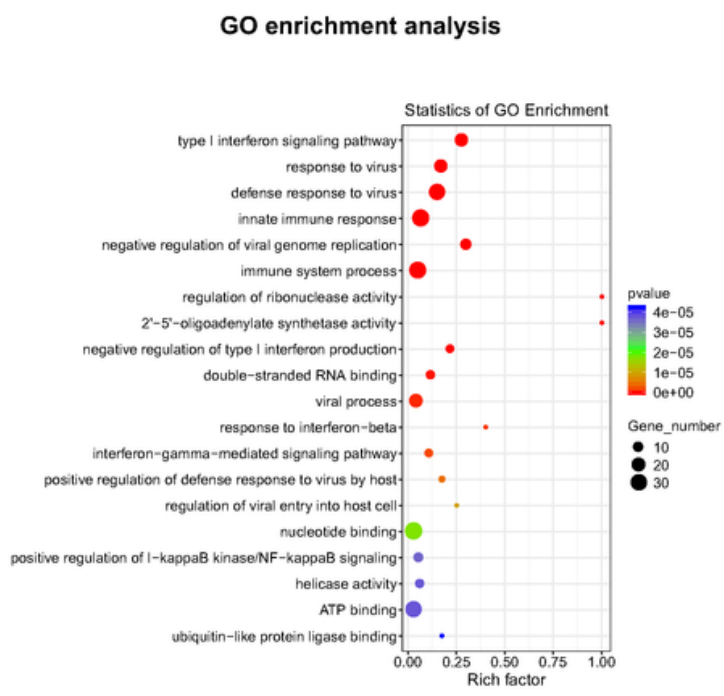

C

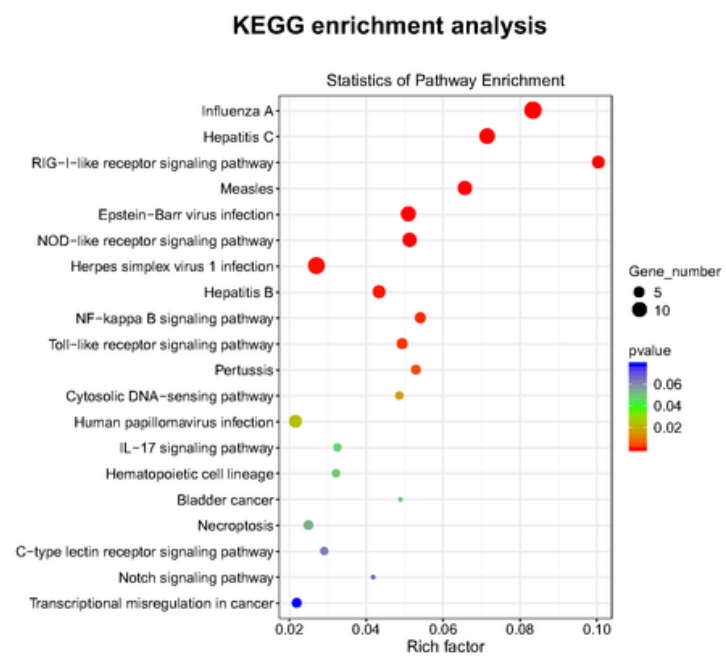

\section{Figure 5}

The impacts of NSUN5 expression on immune-relevant expression profiles in GBM cells (A) Volcano plots of DEGs from RNA sequencing on U251 cells after transfection of siCtrl and siNSUN5\#2; NSUN5 knockdown was confirmed by Western blot; $(B, C)$ GO and KEGG analyses of enriched pathways on the basis of the DEGs; (D) U251 cells were transfected with siCtrl, siNSUN5\#1 and siNSUN5\#2; The protein levels of cGAS-STING pathway components were detected by Western blot; statistical significance at the level of $\mathrm{ns}>0.10, \mathrm{~ms}=0.05 \sim 0.10, *<0.05, * *<0.01$ and $* \star *<0.001 ; \mathrm{GBM}=$ glioblastoma; $\mathrm{GO}=$ gene ontology; KEGG= Kyoto Encyclopedia for Genes and Genome; DEG=differentially expressed gene 
A

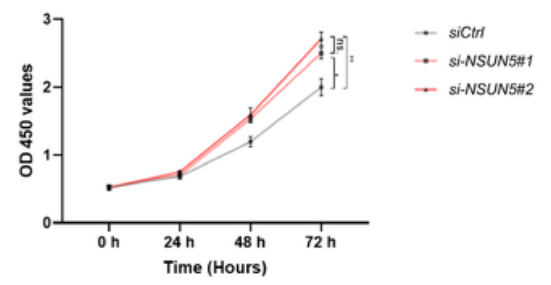

C

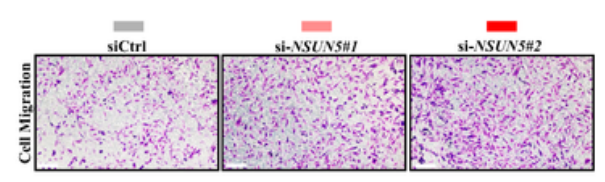

D



B
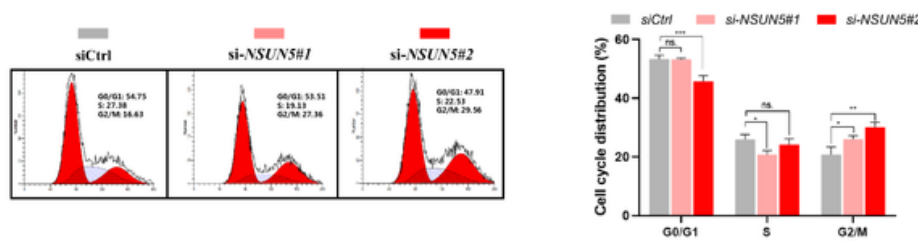

E
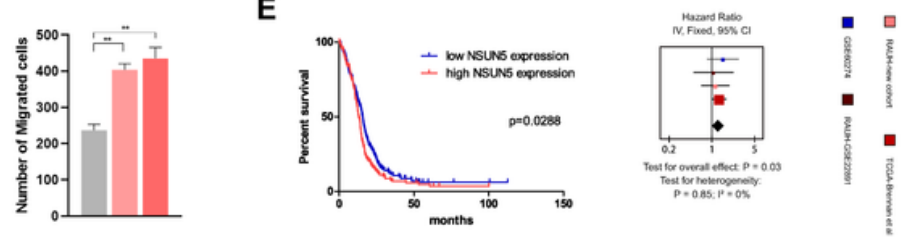

$\mathbf{F}$
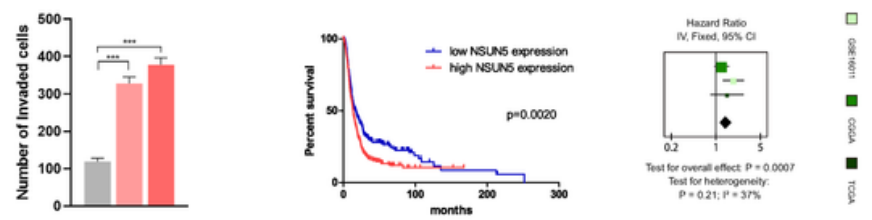

G


I
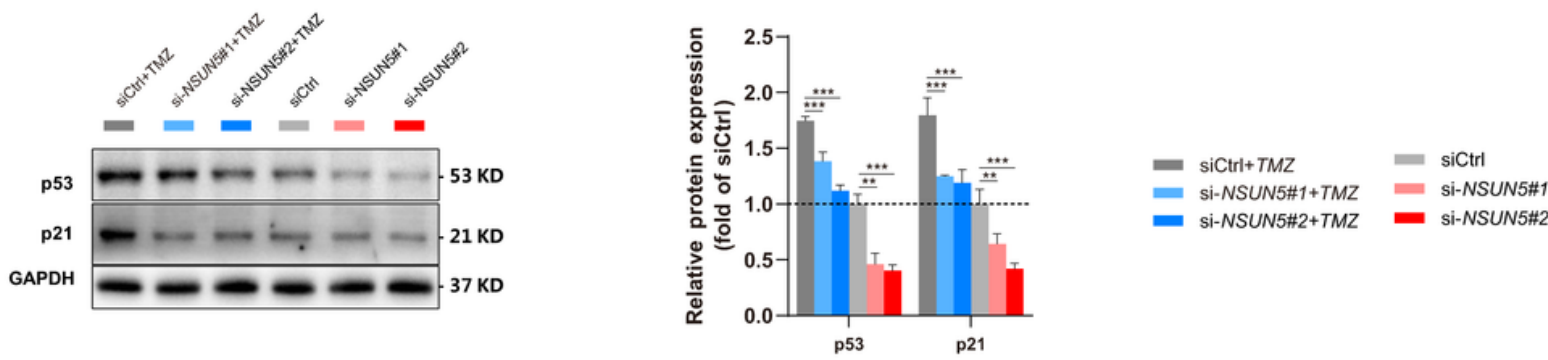

\section{Figure 6}

The impacts of NSUN5 expression on malignant phenotypes and TMZ sensitivity of GBM cells U251 (A) Cell proliferation by CCK-8 assay; (B) Cell cycle by PI-FCS analysis; (C) Cell migration by Transwell chamber assay, scare bar= $200 \mu \mathrm{m}$; (D) Cell invasion by Matrigel chamber assay, scare bar= $200 \mu \mathrm{m} ;(\mathrm{E}-\mathrm{F})$ Survival curves of non-G-CIMP GBMs or IDHwt gliomas of grade II to IV with low vs. high NSUN5 expressions using optimal cutoffs by maxstat R package and forest plots of meta-analysis; (G) IC50 of TMZ treatment by CCK-8 assay; $(\mathrm{H})$ Cell apoptosis by Annexin V-FITC/PI double staining method with or 
without addition of TMZ(200 $\mu \mathrm{M})$; (I) The protein levels of p53 and p21 were detected by Western blot with or without addition of TMZ $(200 \mu \mathrm{M})$; statistical significance at the level of $\mathrm{ns}>0.10, \mathrm{~ms}=$ $0.05 \sim 0.10, *<0.05, * *<0.01$ and $* \star *<0.001 ; \mathrm{TMZ}=$ temozolomide; GBM=glioblastoma; FITC/PI= Annexin Vfluorescein isothiocyanate (FITC)/ Propidium iodide (PI) flow cytometric analysis; PI-FCs= Propidium iodide (PI) flow cytometric analysis

\section{Supplementary Files}

This is a list of supplementary files associated with this preprint. Click to download.

- supplfigs1.pdf

- supplfigs2.pdf

- supplfigs3.pdf

- supplfigs4.pdf

- supplfigs5.pdf

- supplfigs6.pdf

- supplfigs7.pdf

- supplfigs8.pdf

- supplfigs9.pdf

- suppltables1.doc

- suppltables2.xIsx

- suppltables3.xlsx 